\title{
ON THE RELATION BETWEEN \\ GAMES IN EXTENSIVE FORM AND \\ GAMES IN STRATEGIC FORM
}

SimON M. HuTTEGGER

University of California, Irvine

\begin{abstract}
Von Neumann and Morgenstern (1944) claimed that a game in extensive form can always be reduced to a game in strategic form without loss of essential information about the structure of the game. We will evaluate this claim from the point of view of evolutionary game theory. The bottomline is that due to certain generic equilibrium structures of extensive form games they have other dynamical properties than games in strategic form.
\end{abstract}

\section{INTRODUCTION}

John von Neumann and Oskar Morgenstern started their Theory of Games and Economic Behavior by developing so-called games in extensive form. A game in extensive form is an interactive decision problem which is described by a sequence of moves on a game tree. The game starts at a root, where a player or Nature chooses an action (Nature being an additional player used for introducing chance moves). Each action leads to a node where another player chooses. It can also lead to a terminal node where the game ends. At a terminal node, each player receives a payoff which is expressed in terms of a von-Neumann-Morgenstern utility function.

This description of games in extensive form can be formalized in a number of different ways. ${ }^{1}$ There are two concepts which are of particular interest. An information set is a subset of the set of nodes of the game tree where the same player chooses; the nodes in this set cannot be distinguished by the player. This means that the player does not know at which decision node in the information set she is. The second concept is called perfect recall. It means that a player never forgets something that she already knew. Perfect recall is a standard assumption for games in extensive form.

\footnotetext{
${ }^{1}$ There are many excellent introductions to game theory. The interested reader should e.g. consult Binmore (2007) or Fudenberg and Tirole (1991).
} 
An example of a game in extensive form is depicted in Fig.1. It will serve as our main example for this paper. It is Selten's famous Chain Store paradox (Selten, 1978), which is also called the Entry Deterrence game. Player 1 chooses whether to enter a market dominated by a chain store (Player 2). She can either enter $(E)$ or not enter $(N)$. If she enters, then Player 2 can either fight $(F)$ or yield $(Y)$. If Player 1 does not enter, then the status quo gets enshrined. If Player 2 chooses to fight after Player 1 has entered, then both suffer losses, but Player 1 more heavily. If Player 2 chooses to yield, then the players share the market. Notice that each information set is a singleton.

Games in extensive form model the sequence of moves of the players. In strategic form games, a set of strategies is associated with each player. The payoffs to a player then result from strategy combinations. Each player chooses one strategy from her strategy set. The players choose simultaneously. A player's utility function depends on her strategy and the other players' strategies.

Consider Fig. 2. There are two players, each one having two strategies. Their payoff can be given in a payoff table. Each strategy combination results in a payoff for each player. ${ }^{2}$

Von Neumann and Morgenstern (1944) described a way of how games in extensive form can be represented as games in strategic form (see Section 2 below). They also claimed that this can be done without loosing important mathematical information about the game's structure. This claim was rejected by Selten $(1965,1975,1978)$ on grounds of the players' rationality (see also Harsanyi and Selten, 1988).

We will reject the claim on grounds of evolutionary and dynamical considerations. Thus, extensive form games cannot be genuinely reduced to games in strategic form in the sense that the strategic form representation retains all relevant mathematical properties of the extensive form. In this paper I shall develop this argument in more detail. It is based on the topology of Nash equilibria (Section 2) and its dynamical implications (Sections 3 and 4).

\footnotetext{
${ }^{2}$ A more detailed account of games in strategic form can as well be found in standard textbooks.
} 


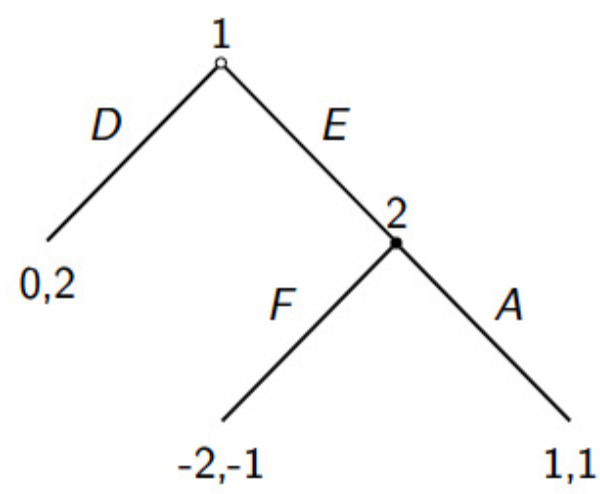

Fig. 1: The Entry-Deterrence game

\begin{tabular}{|c|c|c|}
\hline & $L$ & $R$ \\
\hline$U$ & $a_{11}, b_{11}$ & $a_{12}, b_{12}$ \\
\hline$D$ & $a_{21}, b_{21}$ & $a_{22}, b_{22}$ \\
\hline
\end{tabular}

Fig. 2: A two-player two-strategy game in strategic form. The entries of the table give the payoffs to the row player and to the column player, respectively.

\section{The Topology OF NASH EQUILIBRIA}

Suppose we are given a game in extensive form. Consider all information sets of a player. A pure strategy is a choice of action at each of those information sets. A players' set of (pure) strategies is given by all the different ways she can choose at each of her information sets. We can rephrase this by saying that a pure strategy of a player prescribes the choice of a specific action at each of the players' information sets. It is as if the player commits to choosing at her information sets in a particular way before the game starts.

Defining the players' strategies in this way yields a strategic form of the extensive form game. Strategies are based on actions at information sets, and payoffs can also be calculated by using the extensive form payoffs.

This is the strategic form of an extensive form game as it was described by von Neumann and Morgenstern (1944). There are other, related, strategic forms as well. Sometimes, two different strategies can be regarded as equivalent if they describe the same probability distribution over terminal nodes. Behavior strategies are another way to represent extensive 
form games. A behavior strategy is given by a probability distribution over actions at an information set. This can, for instance, be interpreted as regarding each player as consisting of a number of agents - one for each of the player's information sets.

The Entry Deterrence game provides a convenient, though somewhat atypical example (due to the fact that there are only two moves and each information set is a singleton). In the standard strategic form of the entry deterrence game, each player has two strategies (see Fig. 3).

\begin{tabular}{|c|c|c|}
\hline & $F$ & $A$ \\
\hline$D$ & 0,2 & 0,2 \\
\hline$E$ & $-2,-1$ & 1,1 \\
\hline
\end{tabular}

Fig. 3: The strategic form of the Entry Deterrence game.

In order to be able to point out certain differences between games in extensive form and games in strategic form we have to introduce the concept of Nash equilibria. A Nash equilibrium is a combination of strategies such that a player cannot gain by unilaterally deviating. ${ }^{3}$

Notice that the structure of Nash equilibria in the strategic form of the Entry Deterrence game is quite peculiar. There is one Nash equilibrium where Player 1 enters and Player 2 yields. This is also the subgame perfect Nash equilibrium of the game. ${ }^{4}$ But there is also a set of Nash equilibria, which is given by Player 1 choosing not to enter and Player 2 choosing to fight with sufficiently high probability. This defines a line of Nash equilibria. Thus, the subgame perfect Nash equilibrium is isolated, while none of the other Nash equilibria is isolated.

Are non-isolated Nash equilibria typical for strategic form games? The answer to this question is no. Take the strategic form of Figure 2 as an example. Non-isolated Nash equilibria like the ones in the Chain-Store game are associated with a player receiving the same payoff for at least

\footnotetext{
${ }^{3}$ This means that a player attains her maximum payoff by choosing her part of a Nash equilibrium given that the other players choose their part of it. There may be other strategies that pay off equally well. If there are no such alternative strategies for any of the players, then the Nash equilibrium is strict.

${ }^{4}$ A Nash equilibrium is subgame perfect if it is a Nash equilibrium for each subgame of the extensive form of a game. For example, the part of the game tree of the Entry Deterrence game starting with the node where Player 2 chooses is a subgame. Subgame perfectness prescribes optimal choices even at information sets that are unreached in equilibrium.
} 
two different choices of the other player. By perturbing the payoffs just slightly, the payoffs will be unequal. This argument can be developed precisely (Wu and Jiang, 1962). One way to put it is this: The payoff configurations which allow for non-isolated Nash equilibria are degenerate, i.e. they have measure zero in the space of all payoff configurations.

Thus, if we just look at games in strategic form we are led to think of Nash equilibria as being typcially isolated. Sets of Nash equilibria with other topological properties are naturally discarded as non-generic. This does not hold for games in extensive form, however. Take the Entry Deterrence game as an example. Perturbing the payoffs at the terminal nodes still results in one subgame perfect Nash equilibrium and a continuum of Nash equilibria where Player 1 does not enter the market and Player 2 fights with sufficiently high probability. The reason for this is that Player 2's decision node is not reached because Player 1 decides not to enter the market. This puts less constraints on Player 2's choice of action since in equilibrium she is never actually called to make a choice.

This is a feature of extensive form games in general (see Fudenberg and Tirole, 1991). By choosing particular actions, players forfeit parts of the game tree. This has profound consequences for the topology of the Nash equilibria of the corresponding strategic form games (the standard strategic form, the reduced strategic form and the representation in terms of behavior strategies as well). The payoff configurations will in general be confined to a subspace of the full space of payoff configurations of the strategic form. In the Entry Deterrence game, for instance, there are six payoff parameters, while the corresponding strategic form structure has eight payoff parameters. Payoffs of the extensive form are thus constrained to lie in a subspace of the corresponding strategic form payoff space. This fact allows for the genericity of Nash equilibria which are not isolated. Continua of Nash equilibria such as the one in the Entry Deterrence game are typical of games in extensive form, while they can be ignored for strategic form games.

But does this difference in the topology of Nash equilibrium sets have any important consequences? It does indeed when we move to evolutionary game theory. 


\section{STRUCTURAL STABILITY OF DYNAMICAL SYSTEMS}

Let us introduce some important concepts from dynamical systems theory first. A dynamical system takes place in a state space $X$ which is usually some subset of $n$-dimensional real space. (It can also be a Banach space, a metric space, or a $n$-dimensional manifold.) Furthermore, there is a deterministic rule for how states in $X$ change their position over time; i.e. if $x$ is in $X$ then $T(x, t)$ yields the state $x$ evolves to according to the function $T$ after $t$ units of time.

We shall mostly be concerned with dynamical systems as given by a system of (ordinary) differential equations:

$$
d x / d t=f(x)
$$

Here, the function $f$ describes the rate of change relative to time for each state $x$ in $X$. The corresponding deterministic rule $x(t)$ satisfies the condition $d x(t) / d t=f(x(t))$; i.e., the rate of change of the function agrees with the system of differential equations given by $f . x(t)$ is also called the solution orbit or simply the orbit of the system of differential equations. ${ }^{5}$

$x$ is a rest point if $f(x)=0$. Hence there is no change at a rest point $x$. If $x$ is a rest point, then $x$ is Liapunov stable if all nearby solutions stay nearby. If, in addition, nearby solutions converge to $x$, then $x$ is called asymptotically stable. In general, a rest point $x$ is an attractor if there exists a neighborhood $U$ of $x$ such that all orbits starting in $U$ converge to $x$. Similar definitions exist for sets of points other than rest points, such as periodic orbits. For the purposes of this paper, considering rest points will be sufficient.

A well known example of a dynamical system for game theory is the replicator dynamics, which models interactions between strategy types as a selection process (Hofbauer and Sigmund, 1998). The replicator dynamics describes changes in the proportion of a strategy type over time. The rate of change in the frequency of strategy type $i$ is given by $i$ 's current frequency and the difference between its payoff and the average payoff in the population. Thus, below-average types decrease in frequency while aboveaverage types increase in frequency.

As an example we may consider the replicator dynamics of the Entry Deterrence game. Fig. 4 shows a sketch of the solution orbits for a two-

\footnotetext{
${ }^{5}$ There are a number of comprehensive textbooks on dynamical systems and ordinary differential equations like, e.g., Hirsch and Smale (1974)
} 
population replicator dynamics, where one population corresponds to Player 1 of the Entry Deterrence game and the second population corresponds to Player 2.

A basic proposition of evolutionary game theory states that each Nash equilibrium of the game is a rest point of the corresponding replicator dynamics. This can also be seen in Fig.4, where the Nash equilibria are a subset of the set of rest points. The property of being a Nash equilibrium alone does not determine the stability properties of the corresponding rest point. In the Entry Deterrence game, the subgame perfect Nash equilibrium $(E, Y)$ is an asymptotically stable rest point of the corresponding replicator dynamics. Points on the line of Nash equilibria are Liapunov stable, except the point where $p=1 / 3$. It is also important to notice that the line of Nash equilibria attracts an open set of initial conditions.

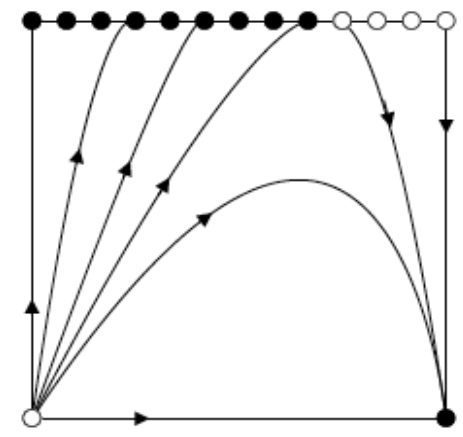

Fig. 4: Replicator dynamics of the Entry Deterrence game. Black dots indicate Nash equilibria. White dots indicate rest points which are not Nash equilibria.

Structural stability is a particularly important concept for dynamical systems. Informally, a dynamical system is structurally stable if all nearby dynamical systems are topologically equivalent. If we consider the dynamical system given by the function $f$, then $g$ is nearby if the values $g(x)$ are sufficiently close to the values $f(x)$ and if the same holds for the partial (and perhaps higher order partial) derivatives of $f$ and $g$. Two dynamical systems are topologically equivalent if there exists a homeomorphism (i.e. a continuous bijective function with continuous inverse) which maps the orbits of the system given by $f$ on the orbits of the system given by $g$ such that the direction of time is preserved (Kuznetsov, 2004). That is to say, two topologically equivalent systems have the same qualitative behavior; e.g., they have the same number of rest points or cycles of the same stability types.

It is important to know what would happen if we changed the specification of the dynamics just a little. After all, we may never be certain 
whether our specification of the dynamics is completely accurate. For a structurally stable system, sufficiently small perturbations will result in the same qualitative dynamical behavior. In this case, even if our specification is not completely accurate, the predictions of the dynamical model are the same up to topological equivalence. Perturbing a structurally unstable system will result in qualitative differences, however. This has important consequences for the relation between the extensive and the strategic form of a game.

\section{STRUCTURAL STABILITY AND DYNAMIC GAMES}

It is important to note that a continuum of rest points implies that the dynamical system under study is structurally unstable. To be somewhat more specific, the existence of a continuum of rest points implies the existence of zero eigenvalues of the Jacobian matrix of the system of differential equations evaluated at one of the rest points in the continuum. The rest point is non-hyperbolic. Zero eigenvalues indicate here that there is no change along the continuum of rest points. But zero eigenvalues will not persist under perturbations of the system. They will turn into positive or negative eigenvalues.

Let us consider the replicator dynamics of the Entry Deterrence game. The existence of a continuum of rest points $M$ where Player 1 chooses not to enter and Player 2 mixes between fighting and yielding allows us to conclude that the replicator dynamics of this game is not structurally stable. Thus, small perturbations of the replicator equations will change the qualitative behavior of the dynamics close to $M$. In principle, a number of different changes are conceivable. $M$ might collapse into a pair of rest points, one of them asymptotically stable and the other one unstable; or their might be no rest point close to $\mathrm{M}$ for the perturbation at all. (See Hofbauer and Huttegger, 2008; cf. Fig.5). Other scenarios are also possible. What happens under perturbations will depend on the properties of the perturbation terms. 

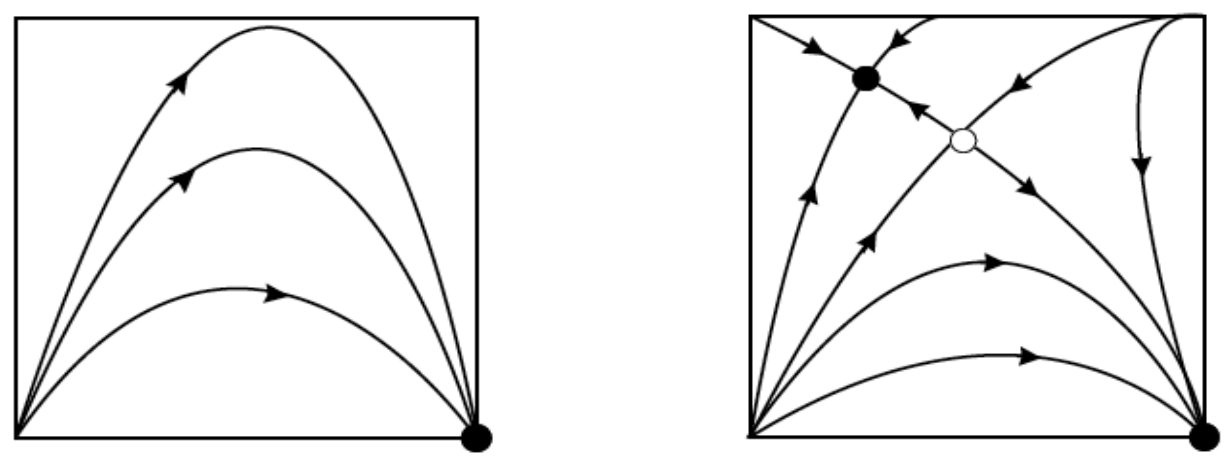

Fig. 5: Possible perturbations of the replicator dynamics for the Entry Deterrence game.

Let us note this point concerning extensive form games and the replicator dynamics. Since continua of Nash equilibria are typical of games in extensive form (in the sense that they persist under payoff perturbations), and since Nash equilibria are rest points of the replicator dynamics, the replicator dynamics of extensive form games will typically be structurally unstable. Is the same true for games in strategic form?

I intend to give an answer to this question in terms of the muli-population replicator dynamics (i.e., one population for each player position). A Nash equilibrium is a solution to a system of inequalities: Each strategy in a Nash equilibrium has to be a best response to the other players' strategies. It can be shown that being a solution to this system of inequalities implies that the solution is also a zero of a system of equations which is the the multi-population replicator dynamics (Ritzberger, 2002, Section 6.5). This is just a restatement of the fact that Nash equilibria are rest points of the replicator equations. Given this fact, a Nash equilibrium $s$ is defined to be regular if the Jacobian matrix $J$ of the replicator equations evaluated at this point is non-singular. This is equivalent to saying that $J$ has no eigenvalues with zero real part or that the rest point corresponding to $s$ is hyperbolic. Hyperbolicity of a rest point implies that the replicator dynamics close to this rest point is structurally stable (Kuznetsov, 2004).

The subgame perfect equilibrium of the Entry-Deterrence game is an example of a regular Nash equilibrium. Nash equilibria in the continuum of Nash equilibria are not hyperbolic, however. But there is a sense in which regular Nash equilibria are typical for games in strategic form. It can be proven that for almost all games in strategic form all Nash equilibria are regular. ${ }^{6}$ Again, this can be understood in terms of payoff perturbations. If we have a game in strategic form with a Nash equilibrium that is not regul-

\footnotetext{
${ }^{6}$ For proofs see Harsanyi (1973), Ritzberger (1994), or Wilson (1971).
} 
ar, then an arbitrarily small perturbation of its payoffs yields a game with no such Nash equilibrium.

Thus we can note the following for games in strategic form. Typically, the replicator dynamics close to a Nash equilibrium is structurally stable. Small perturbations of the dynamics will not alter its qualitative behavior near Nash equilibria.

In this sense, the replicator dynamics and games in strategic form match each other in a way that is not the case for the replicator dynamics and games in extensive form. For the latter, the replicator dynamics will typically not be structurally stable close to Nash equilibria. This allows us to conclude that there is a significant mathematical difference between games in extensive form and games in strategic form from the viewpoint of evolutionary game theory.

\section{CONCLUSION}

The considerations above indicate that there is a significant difference between games in extensive form and games in strategic form when we consider the properties of the corresponing replicator dynamics. The replicator dynamics is arguably the central dynamical system of evolutionary game theory. It remains to be seen, however, to what extent this result carries over to other evolutionary dynamics. I suspect that there will be similar results for dynamics which respect the Nash equilibrium structure in the sense that Nash equilibria are rest points of the dynamics.

I would like to emphasize that the differences between the extensive form and the strategic form arises in the dynamic context for a similar reason as they arise in a rational choice context; in an extensive form game information sets may be unreached in equilibrium. This will result in mixed behavior strategies at these information sets and thus in structurally unstable dynamical systems.

\section{REFERENCES}

Binmore, K. (2007). Playing for Real. A Text on Game Theory. Oxford, Oxford University Press.

Fudenberg, D., and J. Tirole (1991). Game Theory. Cambridge, MA, MIT Press.

Harsanyi, J.C. (1973). Oddness of the Number of Equilibrium Points: a New Proof. International Journal of Game Theory 2: pp.235-250. 
Harsanyi, J.C., and R. Selten (1988). A General Theory of Equilibrium Selection in Games. Cambridge, MA, MIT Press.

Hirsch, M., and S. Smale (1974). Differential Equations, Dynamical Systems, and Linear Algebra. Orlando, FL, Academic Press.

Hofbauer, J., and S.M. Huttegger (2008). Feasibility of communication in binary signaling games. Journal of Theoretical Biology 254: pp.843-849.

Hofbauer, J., and K. Sigmund (1998). Evolutionary Games and Population Dynamics. Cambridge, Cambridge University Press.

Kuznetsov, Y.A. (2004). Elements of Applied Bifurcation Theory. New York, Springer, $3^{\text {rd }}$ edition.

von Neumann, J., and O. Morgenstern (1944). Theory of Games and Economic Behavior. Princeton, Princeton University Press.

Ritzberger, K. (1994). The Theory of Normal Form Games from the Differentiable Viewpoint. International Journal of Game Theory 23: pp.201-236.

Ritzberger, K. (2002). Foundations of Non-Cooperative Game Theory. Oxford, Oxford University Press.

Selten, R. (1965). Spieltheoretische Behandlung eines Oligopolmodells mit Nachfrageträgheit I und II. Zeitschrift für die gesammte Staatswissenschaft 121: pp.301-324, 667-689.

Selten, R. (1975). Re-examination of the Perfectness Concept for Equilibrium Points in Extensive Games. International Journal of Game Theory 4: pp.25-55.

Selten, R. (1978). The Chain Store Paradox. Theory and Decision 9: pp.127-159.

Wilson, R. (1971). Computing Equilibria of $n$-person Games. Siam Journal of Applied Mathematics 21: pp.80-87.

Wu, W.-T., and J.-H. Jiang (1962). Essential Equilibrium Points of $n$-person Noncooperative Games. Scientia Sinica 11: pp.1307-1322. 
